\title{
Profile of olaparib in the treatment of advanced ovarian cancer
}

\author{
This article was published in the following Dove Press journal: \\ International Journal of Women's Health \\ 18 April 2016 \\ Number of times this article has been viewed
}

\author{
Dana M Chase ${ }^{1,2}$ \\ Shreya Patel ${ }^{2,3}$ \\ Kristin Shields ${ }^{4}$ \\ 'Division of Gynecologic Oncology, \\ The University of Arizona Cancer \\ Center, ${ }^{2}$ Division of Gynecologic \\ Oncology, ${ }^{3}$ Creighton University \\ School of Medicine, St Joseph's \\ Hospital and Medical Center, \\ Phoenix, AZ, ${ }^{4}$ Department of \\ Trauma, Critical Care, and Acute \\ Care Surgery, Medical College of \\ Wisconsin, Milwaukee, WI, USA
}

Correspondence: Dana M Chase Division of Gynecologic Oncology, 500 West Thomas Road, Suite 600, Phoenix, AZ 850। 3, USA

Tel + I 6024067730

Fax + I 6027980807

Email dana.chase@chw.edu

\begin{abstract}
Olaparib is a poly(ADP-ribose) polymerase inhibitor that received accelerated approval from the US Food and Drug Administration as monotherapy for patients with germline BRCA mutations and ovarian cancer treated with three or more prior lines of chemotherapy. This article summarizes the mechanism of poly(ADP-ribose) polymerase inhibition, therapeutic profile and uses of olaparib, and current and ongoing literature pertaining to olaparib in advanced ovarian cancer.
\end{abstract}

Keywords: olaparib, PARP, ovarian cancer

\section{Introduction}

Olaparib (AZD-2281, trade name Lynparza) is a poly(ADP-ribose) polymerase (PARP) inhibitor approved by US Food and Drug Administration (FDA) as monotherapy for ovarian cancer patients with germline BRCA mutations who have been treated with three or more prior lines of chemotherapy. The FDA concurrently approved the BRACAnalysis CDx ${ }^{\mathrm{TM}}$ (Myriad Genetics) as a companion diagnostic test. The BRACAnalysis CDx ${ }^{\mathrm{TM}}$ tests for deleterious or suspected deleterious variants in BRCA 1/2 using whole blood specimens. PARP $1 / 2$ enzymes repair single-stranded DNA breaks through base excision repair. Unrepaired single-stranded breaks are converted into double-stranded breaks when cells attempt replication or transcription. Doublestranded breaks are normally repaired with homologous recombination. However, up to $50 \%$ of high-grade serous ovarian cancers lack homologous recombination through a combination of germline mutations in BRCA $1 / 2$; silencing via methylation of the, eg, BRCA promoter or Fanconi F; activation of pathway inhibitors; or other mechanisms. ${ }^{1}$ This leaves cells dependent on nonhomologous end joining, an error-prone repair method that leads to genetic instability and cell death. BRCA $1 / 2$ are homologous repair proteins; therefore, cells with BRCA 1/2 mutations are specifically targeted by PARP inhibition. PARP inhibitors also trap PARP1 and 2 enzymes on DNA, leading to complexing, which interferes with DNA replication and causes further cellular damage. ${ }^{2}$ This article reviews the pharmacologic and safety profile of olaparib as well as the effects of BRCA mutations and platinum sensitivity on efficacy and the ongoing research in the use of olaparib for advanced ovarian cancer.

\section{Pharmacokinetics, dose response, and safety}

Olaparib is a rapidly absorbed orally active agent with peak plasma levels attained at 1-3 hours after administration and a mean terminal half-life of 11.9 hours. Phase I pharmacokinetic and pharmacodynamic testing of olaparib in 60 patients with solid tumors, including 21 ovarian cancer patients, established a maximum tolerated dose 
of $400 \mathrm{mg}$ taken twice daily continuously. Toxicities were generally mild and included fatigue and gastrointestinal symptoms, such as nausea, vomiting, taste alteration, and anorexia. ${ }^{3}$ Table 1 demonstrates the frequency of adverse events encountered in clinical trials using olaparib. The most common laboratory abnormalities included increased mean corpuscular volume, decreased hemoglobin, increased creatinine, and decreased neutrophil count. There is a specific warning in the product insert regarding the development of myelodysplastic syndrome (MDS). Of 2,618 patients who had received olaparib at the time of the June 2014 FDA briefing, $21(0.8 \%)$ had reported MDS and/or acute myeloid leukemia. Although the prior administration of platinumbased chemotherapy may predispose patients to the development of MDS/acute myeloid leukemia, this percentage is larger than what would be expected. Olaparib is metabolized via CYP3A, and metabolites are excreted in urine (44\%) and feces $(42 \%){ }^{4}$

The FDA-approved formulation of olaparib is a $50 \mathrm{mg}$ capsule. The achievement of the recommended dose requires administration of eight capsules twice daily. A tablet formulation has also been developed that is available as $100 \mathrm{mg}$ or $150 \mathrm{mg}$, with a recommended dose of $300 \mathrm{mg}$ bid. While the tablet form has increased bioavailability compared to the capsule formation, the efficacy and tolerability of the $300 \mathrm{mg}$ bid tablet dose is comparable to those of the $400 \mathrm{mg}$ bid capsule formulation. ${ }^{5}$ The recommended dosing regimen for olaparib is $400 \mathrm{mg}$ twice daily. A prospective multicenter Phase II study of patients with recurrent ovarian cancer and germline BRCA $1 / 2$ mutations and one or more prior lines of chemotherapy evaluated the objective response rate (ORR) in patients treated with continuous olaparib $400 \mathrm{mg}$ twice daily (maximum tolerated dose) or $100 \mathrm{mg}$ twice daily (dose of first clinical response). The ORR was 33\% (eleven of 33 patients, $95 \%$ confidence interval [CI] 20-51) in the $400 \mathrm{mg}$ group and $13 \%$ (three of 24 patients, $95 \%$ CI 4-31) in the $100 \mathrm{mg}$ group. There was an unscheduled interim analysis and protocol amendment allowing patients in the $100 \mathrm{mg}$ group to increase dose to $400 \mathrm{mg}$ twice daily due to an observed increased frequency of early progression in the lower dose group. The median progression-free survival was 5.8 months ( $95 \%$ CI $2.8-10.6$ ) in the $400 \mathrm{mg}$ group versus 1.9 months (95\% CI 1.8-3.6) in the $100 \mathrm{mg}$ group. ${ }^{6}$

The effect of food on the rate and amount of absorption of olaparib was studied in an open-label randomized trial of 32 patients. Compared to fasting state, administration after a standard meal or high-fat meal increased exposure to olaparib by $\sim 20 \%$, which was not considered

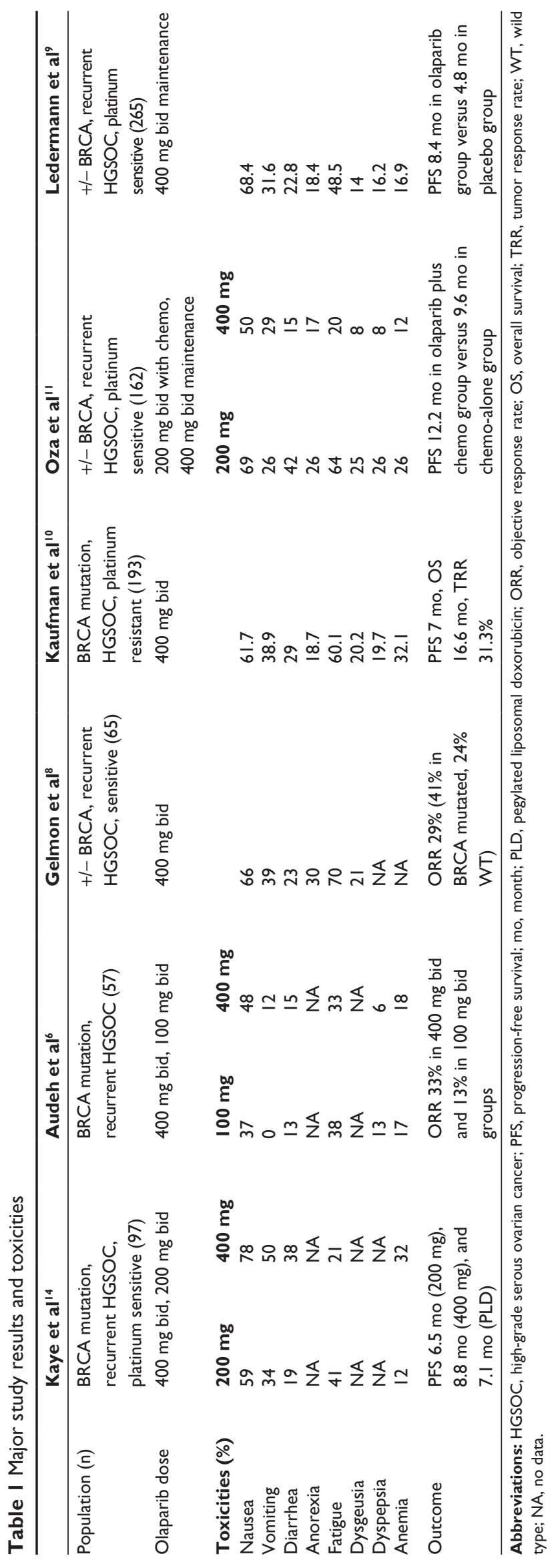


clinically significant. Feeding also increased the rate of absorption. ${ }^{7}$

\section{Efficacy in patients without BRCA mutations}

Olaparib has also demonstrated efficacy in patients without BRCA mutations. Gelmon et al conducted a multicenter, nonrandomized Phase II study that included 63 women with advanced high-grade serous ovarian cancer. The patients were treated with olaparib $400 \mathrm{mg}$ twice daily. The ORR was $41 \%$ (seven of $17,95 \%$ CI 22-64) for patients with BRCA $1 / 2$ mutations and $24 \%$ (eleven of $46,95 \%$ CI 14-38) for patients without BRCA $1 / 2$ mutations. In this study, patients with platinum-sensitive disease had ORR 50\% in the BRCA $1 / 2$ mutation negative cohort versus $60 \%$ in the cohort with mutation. This contrasted with the platinumresistant population, where the ORR was $33 \%$ in patients with BRCA $1 / 2$ mutation and $4 \%$ in the BRCA $1 / 2$ mutation negative cohort. ${ }^{8}$

\section{Efficacy of olaparib in platinum- sensitive and platinum-resistant diseases}

Maintenance therapy in platinum-sensitive patients improved progression-free survival in comparison to placebo in a randomized, double blind, Phase II trial by Ledermann et al. Two hundred and sixty-five patients with recurrent ovarian cancer who had at least two prior courses of platinum-based chemotherapy and an objective response to the most recent regimen were randomized to olaparib $400 \mathrm{mg}$ twice daily or matching placebo until progression. The progression-free survival was 8.4 months with olaparib compared to 4.8 months with placebo (hazard ratio [HR] $0.35,95 \%$ CI $0.25-0.49 ; P<0.001$ ). The patient's quality of life, as measured by Functional Assessment of Cancer Therapy - Ovarian (FACT-O) and variables derived from the FACT-O, was not different between olaparib and placebo groups. Most patients reported a best treatment outcome index as "improved" or "no change" during treatment, supporting the conclusion that there was no negative impact on the patient's quality of life during treatment. ${ }^{9}$

Efficacy in platinum resistance was investigated in a multicenter Phase II trial by Kaufman et al. A study population of 298 patients with platinum-resistant cancers and a germline BRCA 1/2 mutation (including 193 patients with ovarian, primary peritoneal, or fallopian tube cancers) was administered olaparib $400 \mathrm{mg}$ twice daily until disease progression. These patients had a mean of 4.3 prior regimens. The tumor response rate was $26.2 \%$ overall (95\% CI $21.3-31.6)$, and the response rate for ovarian cancer patients was $31.1 \%$ (95\% CI 24.6-38.1). The median duration of response was 225 days, and the median progression-free survival was 7 months. $^{10}$

\section{Olaparib in combination with chemotherapy}

It has been proposed that olaparib could potentiate the effect of DNA-damaging chemotherapy. Oza et al conducted a randomized, open-label, Phase II study of 162 patients with recurrent, platinum-sensitive, high-grade serous ovarian cancers who had received up to three prior regimens of platinum-based chemotherapy. Patients received olaparib $200 \mathrm{mg}$ twice daily plus paclitaxel $165 \mathrm{mg} / \mathrm{m}^{2}$ and carboplatin AUC4 followed by olaparib $400 \mathrm{mg}$ twice daily maintenance versus carboplatin and paclitaxel alone. Patients in the olaparib plus chemotherapy group had a longer progression-free survival (12.2 versus 9.6 months for chemotherapy alone, HR 0.51, 95\% CI 0.34-0.77; $P=0.0012$ ). This benefit was more pronounced in patients with known BRCA mutation (HR 0.21, 95\% CI 0.08-0.55; $P=0.0015) .{ }^{11}$

The addition of antiangiogenic agents to olaparib has also been studied. An open-label comparison of olaparib $400 \mathrm{mg}$ twice daily or olaparib $200 \mathrm{mg}$ twice daily with cediranib $30 \mathrm{mg}$ daily in patients with recurrent, high-grade, platinumsensitive serous ovarian cancer or BRCA-related ovarian cancer evaluated 90 patients, stratified by BRCA status. Progression-free survival was 17.7 months in the group receiving cediranib versus 9 months in the olaparib-only group. (HR 0.42, 95\% CI 0.23-0.76; $P=0.005$ ). However, toxicity was higher in the combination group than in the olaparib-alone group (27\% versus 11\% fatigue, 41\% versus $0 \%$ hypertension, and $23 \%$ versus $0 \%$ diarrhea), and $75 \%$ of the combination group required dose reduction. ${ }^{12}$ A small Phase I study investigated increasing doses of olaparib in combination with bevacizumab $10 \mathrm{mg} / \mathrm{kg}$ every 2 weeks. Adverse events were generally mild, and the most frequent events were fatigue and nausea. Significant adverse events (small bowel obstruction with perforation) were attributed to bevacizumab, rather than olaparib. ${ }^{13}$

\section{Efficacy in recurrent ovarian cancer compared to pegylated liposomal doxorubicin}

An open-label, randomized trial of women with ovarian, primary peritoneal, or fallopian tube cancers that recurred or progressed within 12 months of the most recent platinumbased chemotherapy investigated olaparib at $200-400 \mathrm{mg}$ 
twice daily versus intravenous pegylated liposomal doxorubicin (PLD) $50 \mathrm{mg} / \mathrm{m}^{2}$ every 28 days. The trial enrolled 97 patients and allowed crossover from PLD group to $400 \mathrm{mg}$ olaparib group in the event of disease progression. In the $200 \mathrm{mg}$ olaparib, $400 \mathrm{mg}$ olaparib, and PLD groups, the median progression-free survival was 6.5 months $(95 \%$ CI 5.5-10.1), 8.8 months (95\% CI 5.4-9.2), and 7.1 months (95\% CI 3.7-10.7 months), respectively. The ORRs were $25 \%, 31 \%$, and $18 \%$, respectively. There was no statistically significant difference between the olaparib or PLD groups in terms of either progression-free survival or ORR. Grade 3 to 4 treatment associated adverse events were more common in the PLD group. Improvement in the patient's quality of life as measured by FACT-O was significantly increased in the olaparib group versus the PLD group (odds ratio 7.23, 95\% CI 1.09-143.3; $P=0.039) .{ }^{14}$

\section{Long-term safety and efficacy}

Van der Noll et al collected data on the long-term safety and efficacy of olaparib in a Phase I cohort of 21 patients who had received olaparib in combination with carboplatin and/or paclitaxel. Patients continued olaparib monotherapy (400 mg bid) after the Phase I combination study if they had completed six or more cycles of combination therapy, had no evidence of disease progression, and had significant toxicity from the combination therapy. Bone marrow suppression, fatigue, pain, and nausea decreased over time after combination therapy was discontinued. Gastrointestinal toxicities, such as gastritis, esophagitis, and dyspepsia, increased in frequency with longer duration use of olaparib, but were reduced with proton pump inhibitors. Response to therapy was diuturnal, with the median duration of treatment 52 weeks. The best overall response was $43 \%$ complete response, $22 \%$ partial response, $29 \%$ stable disease, $5 \%$ progressive disease, and $5 \%$ not evaluable. ${ }^{15}$

\section{Ongoing research}

SOLO-1 and SOLO-2 are multicenter, double-blind studies of olaparib $300 \mathrm{mg}$ bid versus placebo in patients with high-grade serous or endometrioid ovarian, fallopian tube, or primary peritoneal cancer. Both SOLO-1 and SOLO-2 have primary outcomes of progression-free survival by response evaluation criteria in solid tumors (RECIST) criteria in olaparib $300 \mathrm{mg}$ twice daily monotherapy maintenance compared to placebo. The study population of SOLO-1 is BRCAmutated high-risk advanced ovarian cancer patients who achieved complete or partial response with first-line platinum chemotherapy, whereas the population of SOLO-2 is BRCAmutated relapsed ovarian cancer patients who achieved complete or partial response with platinum chemotherapy. In SOLO-1, the patients will receive olaparib versus placebo for up to 2 years or until objective response by RECIST criteria and the treatment may continue beyond 2 years for those with stable disease or progression. Patients in SOLO-2 will receive olaparib versus placebo until progression by RECIST criteria or as long as they are benefiting from treatment and do not meet other criteria for discontinuation. ${ }^{16,17}$

The current FDA approval for olaparib is for patients who have received three or more prior lines of chemotherapy. A recent study by Marques et al investigated whether the administration of chemotherapy affected intratumoral PARP expression. Tissue samples were obtained from three cohorts, totaling 313 high-grade serous ovarian cancers. PARP1 expression was measured by immunohistochemistry and Western blot protein analysis. Samples from patients who had undergone chemotherapy had significantly lower protein expression than chemotherapy-naïve tumor samples. Of 15 samples from patients which had both pre- and postchemotherapy (carboplatin and paclitaxel), six had absent PARP1 expression and eight had reduced PARP1 levels postchemotherapy. The clinical significance of this change in PARP1 expression is unknown; however, the dynamic interaction of PARP1 and chemotherapy should be taken into consideration in future studies. ${ }^{18}$ It is possible that the use of olaparib before exposure to multiple lines of chemotherapy would be a more effective use.

\section{Conclusion}

Olaparib is a well-tolerated oral chemotherapeutic agent that has FDA approval for the treatment of ovarian cancer in patients with germline BRCA $1 / 2$ mutations who have received three or more prior lines of chemotherapy. In the future, development of assays for function of homologous recombination may help to identify patients without a germline BRCA 1/2 mutation who would also benefit from treatment with olaparib. Further studies will also help delineate the optimal timing and setting of olaparib administration.

\section{Disclosure}

The authors report no conflicts of interest in this work.

\section{References}

1. Lee JM, Ledermann JA, Kohn EC. PARP inhibitors for BRCA1/2 mutation-associated and BRCA-like malignancies. Ann Oncol. 2014; 25(1):32-40.

2. Liu JF, Konstantinopoulos PA, Matulonis UA. PARP inhibitors in ovarian cancer: current status and future promise. Gynecol Oncol. 2014; 133(2):362-369.

3. Fong PC, Boss DS, Yap TA, et al. Inhibition of poly (ADP-ribose) polymerase in tumors from BRCA carriers. $N$ Engl J Med. 2009;361: 123-134. 
4. FDA. FDA Briefing Document Oncologic Drugs Advisory Committee Meeting. Available from: www.fda.gov. Accessed August 28, 2015.

5. Mateo J, Friedlander M, Sessa C, et al. Administration of continuous/intermittent olaparib in ovarian cancer patients with a germline BRCA1/2 mutation to determine an optimal dosing schedule for the tablet formulation. Eur J Cancer. 2013;49:S161.

6. Audeh MW, Carmichael J, Penson RT, et al. Oral poly(ADP-ribose) polymerase inhibitor olaparib in patients with BRCA1 or BRCA2 mutations and recurrent ovarian cancer: a proof-of-concept trial. Lancet 2010;376(9737):245-251.

7. Rolfo C, Swaisland H, Leunen K, et al. Effect of food on the pharmacokinetics of olaparib after oral dosing of the capsule formulation in patients with advanced solid tumors. Adv Ther. 2015;32:510-522.

8. Gelmon KA, Tischkowitz M, Mackay H, et al. Olaparib in patients with recurrent high-grade serous or poorly differentiated ovarian carcinoma or triple-negative breast cancer: a phase 2, multicentre, open-label, non-randomised study. Lancet Oncol. 2011;12(9):852-861.

9. Ledermann J, Harter P, Gourley C, et al. Olaparib maintenance therapy in patients with platinum-sensitive relapsed serous ovarian cancer: a preplanned retrospective analysis of outcomes by BRCA status in a randomised phase 2 trial. Lancet Oncol. 2014;15(8):852-861.

10. Kaufman B, Shapira-Frommer R, Schmutzler RK, et al. Olaparib monotherapy in patients with advanced cancer and a germline BRCA1/2 mutation. J Clin Oncol. 2015;33(3):244-250.

11. Oza AM, Cibula D, Benzaquen AO, et al. Olaparib combined with chemotherapy for recurrent platinum-sensitive ovarian cancer: a randomised phase 2 trial. Lancet Oncol. 2015;16(1):87-97.
12. Liu JF, Barry WT, Birrer M, et al. Combination cediranib and olaparib versus olaparib alone for women with recurrent platinum-sensitive ovarian cancer: a randomized phase 2 study. Lancet Oncol. 2014;15(11): 1207-1214.

13. Dean E, Middleton MR, Pwint T, et al. Phase I study to assess the safety and tolerability of olaparib in combination with bevacizumab in patients with advanced solid tumours. Br J Cancer. 2012;106:468-474.

14. Kaye SB, Lubinski J, Ang JE, et al. Phase II, open-label, randomized, multicenter study comparing the efficacy and safety of olaparib, a poly (ADP-ribose) polymerase inhibitor, and pegylated liposomal doxorubicin in patients with BRCA 1 or BRCA 2 mutations and recurrent ovarian cancer. J Clin Oncol. 2012;30(4):372-379.

15. Van der Noll R, Marchetti S, Steeghs N, et al. Long-term safety and anti-tumour activity of olaparib monotherapy after combination with carboplatin and paclitaxel in patients with advance breast, ovarian, or fallopian tube cancer. Br J Cancer. 2015;113:396-402.

16. Clinicaltrials.gov. Olaparib Maintenance Monotherapy in Patients With BRCA Mutated Ovarian Cancer Following First Line Platinum Based Chemotherapy. (SOLO-1). Available from: www.clinicaltrials.gov. Accessed August 21, 2015.

17. Clinicaltrials.gov. Olaparib Treatment in BRCA Mutated Ovarian Cancer Patients After Complete or Partial Response to Platinum Chemotherapy. Available from: www.clinicaltrials.gov. Accessed August 21, 2015.

18. Marques M, Beauchamp M-C, Fleury H, et al. Chemotherapy reduces PARP1 in cancers of the ovary: implications for future clinical trials involving PARP inhibitors. BMC Med. 2015;13:217.
International Journal of Women's Health

\section{Publish your work in this journal}

The International Journal of Women's Health is an international, peerreviewed open-access journal publishing original research, reports, editorials, reviews and commentaries on all aspects of women's healthcare including gynecology, obstetrics, and breast cancer. The manuscript management system is completely online and includes

\section{Dovepress}

a very quick and fair peer-review system, which is all easy to use. Visit http://www.dovepress.com/testimonials.php to read real quotes from published authors. 